\title{
UNA APLICACIÓN PEDAGÓGICA DESDE LA PERSPECTIVA DE LA ANTROPOLOGÍA DE LOS CUIDADOS: PROGRAMA DE PREVENCIÓN DE E.T.S Y EMBARAZOS NO DESEADOS EN ADOLESCENTES
}

\author{
Laura Jiménez Ruiz, Miguel Botella Santamaría, Esther Ferrero Lozano, \\ Sergio Trigueros Jiménez, Dolores Seguí Mengual, Noelia Lledó Ivorra, Cristina Espinós Juan \\ Alumnas/os de Pedagogía aplicada a los cuidados (Asignatura optativa del vigente plan de estudios de \\ D.E de la E.U de Enfermería de la Universidad de Alicante.
}

\section{RESUMEN}

El objetivo de nuestro estudio ha sido aumentar conocimientos y corregir errores, sobre el uso de métodos anticonceptivos y prevención de E.T.S. en una muestra de 30 alumnos de $3^{\circ}$ de E.S.O. de un instituto de la provincia de Alicante. Pensamos que ante una necesidad tan evidente podíamos obtener resultados satisfactorios si utilizábamos una pedagogía participativa que atrajese a los alumnos. De este modo, por medio del paradigma sociocrítico conseguimos implicar a la muestra para que tomasen conciencia de sus propias necesidades.

Se impartieron charlas a los alumnos sobre distintos temas de interés que más tarde serían evaluados. La metodología utilizada fue el test-retest. Se distribuyó un primer cuestionario antes de la primera charla para adaptar nuestra ponencia a las necesidades de la muestra, y un segundo test, de iguales características al primero, después de la última charla para evaluar los conocimientos adquiridos.

Los alumnos tenían mayores problemas en aspectos como qué es la sexualidad, dónde acudir para informarnos sobre sexualidad, diferencia entre sexualidad y reproducción, dónde comprar preservativos, qué hacer ante la ruptura de un preservativo, utilización de lubricantes, vías de transmisión de las E.T.S., etc. En estos aspectos se consiguió un porcentaje de éxito en base a nuestros objetivos considerablemente elevado.

Palabras clave: Educación para la Salud, pedagogía aplicada a los cuidados, antropología de la educación, educación sexual.

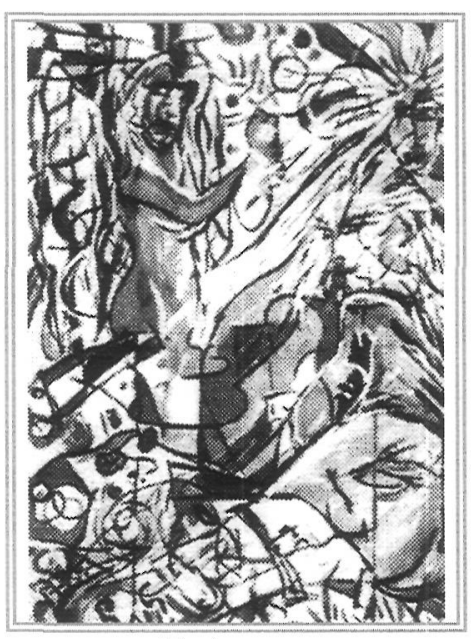

Marí

\section{CONTRACEPTIVE METHODS}

\section{SUMMARY}

The aim of our study was to increase knowledge and correct errors in the use of contraceptive methods and prevention of Sexual Transmission Diseases (STD) in a sample of 30 students in the 3rd year of ESO (GCE)from a school in the Alicante area.

We considered that faced with such an evident need we could obtain very satisfactory results should we use an appealing pedagogical participation which attracted the students. So by means of a critical paradigm we managed to get them involved in the event, concerned of their own needs. Lectures on different interesting topics were given to the students, to be evaluated later on. The method used was that of the test/re-test. A questionnaire was provided before the first lecture so that we could adapt our information to their shown expressed needs; and a second questionnaire of equal characteristics was provided after the last lecture to evaluate their acquired knowledge.

The students' major problems were in knowing: what sexuality means and where to go to get information about it; what is the difference between sexuality and reproduction; where to obtain sheaths; what to do if they get torn; use of lubricants, and means of sexual transmission of diseases (STD). According to our expectation the degree of success was really high. 


\section{INTRODUCCIÓN}

En las últimas décadas se ha observado paulatinamente como la media de edad de la primera relación sexual entre los jóvenes, se iba adelantando poco a poco. En las puertas del año 2.000, la necesidad de educar a los colectivos juveniles en sexualidad se va haciendo cada vez más evidente, especialmente si se tienen en cuenta las Enfermedades de Transmisión Sexual, como el S.I.D.A, que cada día afecta a personas de menor edad e infecta a grupos más variados. Teniendo en cuenta que es una situación modificable mediante la prevención, se comprenderá la importancia de la Educación para la Salud en la población adolescente en base a este tema.

En la Escuela Universitaria de Enfermería de Alicante (E.U.E.), se ha realizado un proyecto en la asignatura Pedagogía Aplicada a los Cuidados de Enfermería (que aparece dentro del curriculum con carácter optativo), que versa sobre la prevención de Enfermedades de Transmisión Sexual (E.T.S.) y embarazos no deseados en adolescentes.

Dicho proyecto se llevó a cabo en una clase de $3^{\circ}$ de E.S.O. del Instituto de Enseñanza Secundaria Leonardo Da Vinci, durante el curso escolar 98/99. Se realizó en el plazo de una semana, por un grupo de trabajo denominado Omega, compuesto por 7 alumnos de Enfermería.

Según estudios previos, la media de edad en la que se mantiene la primera relación sexual se sitúa entre los 17 y los 18 años. Si además tenemos en cuenta que a estas edades se tiende a mantener relaciones sexuales con más de una persona (media de 3,64 para los hombres y 1,907 para las mujeres), se comprenderá la importancia de realizar programas de estas características.

Ya en la primera relación coital, varones y mujeres mantienen un porcentaje similar de sufrir un embarazo no deseado o transmisión de enfermedades, situándose éste en torno al 18\%. Teniendo en cuenta que es un porcentaje bastante elevado, pensamos en la posibilidad de dirigir nuestro proyecto a los adolescentes de entre 14 y 18 años, puesto que los identificamos como población susceptible de riesgo.
Decidimos insistir en el uso del preservativo por ser un método que protege de las E.T.S. y por ser el de mayor elección entre los adolescentes, incluyendo así en nuestro programa un caso práctico sobre la correcta utilización de este contraceptivo.

En otro estudio en el que se recogían datos descriptivos sobre los tratamientos postcoitales administrados por el Centro Joven de Anticoncepción y Sexualidad de Madrid, se observó que la edad de las usuarias que demandaban un tratamiento postcoital oscilaba en su mayoría entre los 13 y los 19 años y además en un $67 \%$ de los casos alegaban como solicitud del tratamiento ruptura del preservativo. Este hecho nos llevó a pensar que deberíamos incluir información sobre qué hacer ante la ruptura de un preservativo o ante un embarazo, lugares a los que pueden acudir y personas que les pueden ayudar.

El objetivo general del estudio fue aumentar conocimientos y corregir errores en la muestra seleccionada, al menos en un 50\% de los casos, sobre el uso de métodos anticonceptivos y prevención de E.T.S.

Teniendo la oportunidad de trabajar con alumnos de edades comprendidas entre 14 y 17 años, tendríamos la certeza de aumentar los conocimientos que se especifican en los objetivos. Así, utilizando un método de Pedagogía participativa y atendiendo a una necesidad tan evidente como la que nos ocupa, pensamos que podríamos obtener una tasa de rentabilidad elevada.

Finalizamos nuestro programa con la narración de un caso real de un embarazo no deseado por parte de la afectada, con el fin de sensibilizar a la muestra en este tema, de modo que viesen el suceso como algo cercano que no solo ocurre en casos excepcionales. A continuación se realizó un debate abierto entre alumnos y ponentes, a fin de que los alumnos pudiesen expresar sus dudas y temores libremente.

Los encuentros fueron grabados en vídeo (aunque con medios caseros) para poder observar las reacciones de los alumnos ante determinados temas y, al mismo tiempo, observar nuestros fallos con el fin de mejorarnos en charlas posteriores. 


\section{MATERIAL Y MÉTODOS}

La población que seleccionamos para el estudio fueron adolescentes de entre 14 y 18 años. La muestra elegida fue un grupo de 30 alumnos de $3^{\circ}$ de E.S.O. con edades comprendidas entre los $14 \mathrm{y}$ 17 años y con una media de edad de 15,4 años. A todos ellos se les explicaron los objetivos de nuestro estudio y los beneficios que podían obtener con nuestras charlas y se les dio la oportunidad de colaborar con entera libertad. No obtuvimos ninguna respuesta negativa a participar en el programa.

La técnica utilizada ha sido el test-retest, mediante el uso de un cuestionario de cumplimentación propia de preguntas en su mayoría abiertas. El primer test se pasó dos días antes de la charla, con la finalidad de detectar las necesidades de la muestra. El segundo cuestionario, de idénticas características al primero, se distribuyó un día después de la charla.

Elegimos un cuestionario no dirigido (sin items), con el propósito de que los alumnos respondiesen libremente para poder detectar con precisión las posibles creencias erróneas y tratar de encaminar la charla atendiendo a las necesidades de la muestra. El tiempo de cumplimentación del mismo era de unos 15 minutos, contando éste con 13 preguntas en las cuales se intenta indagar en tres aspectos fundamentalmente:

1) Sexualidad en general (preguntas de la 1 a la 5).
2) Métodos anticonceptivos (preguntas de la 6 a la 11).

3) Enfermedades de Transmisión Sexual (preguntas 12 y 13$)$.

Además se incluían en el test únicamente dos rasgos sociodemográficos, edad y sexo, puesto que el cuestionario era totalmente anónimo.

Con los resultados obtenidos se ha realizado un estudio estadístico compuesto por la distribución de frecuencias absolutas y porcentajes de ambos cuestionarios por separado, con el fin de poder comparar los datos para su posterior discusión.

A lo largo de nuestro programa, hemos utilizado una línea pedagógica muy participativa basada en las enseñanzas de Freire; en el marco de la Pedagogía activa, tratamos de acercarnos al paradigma socio-crítico, intentando inducir un cambio en los alumnos para que tomen conciencia de sus propios actos y actúen en consecuencia.

\section{RESULTADOS}

\section{RASGOS SOCIODEMOGRÁFICOS:}

A) EDAD: La muestra de nuestro estudio tiene un rango de edades comprendidas entre los 14 y los 17 años, y una media de edad de 15, 4 años.

B) SEXO: El 57\% (17) de la muestra son mujeres, mientras que el $43 \%$ (13) restante son varones.

\section{CUADRO COMPARATIVO DE LAS DOS ENCUESTAS: PREGUNTA RESPUESTA}

PORCENTAJE $1^{\mathrm{a}}$ ENCUESTA
PORCENTAJE $2^{\mathrm{a}}$ ENCUESTA

¿Qué es la sexualidad?

Realizar coito $.63 \%(19)$

Forma de reproducirse . . 17\%

Placer en general. ..... . 7\%

N.S./N.C. . . . . . . 13\%

$13 \%$

$7 \%$

$80 \%(24)$

$0 \%$

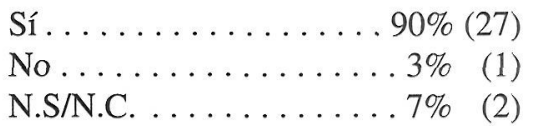

(15)

Con qué frecuencia hablas de sexualidad

Poca............ 50\% (15)

Mucha .......... 30\% (9)

No especifican . . . . . . 20\%
$53 \%(16)$

$40 \%(12)$

$7 \% \quad(2)$ 
PREGUNTA

Con quién hablas de sexualidad.
RESPUESTA

Familia

Amistades/pareja . . . . . . 70\% (21)

No especifican ........ 17\%

PORCENTAJE $1^{\mathrm{a}}$ ENCUESTA

PORCENTAJE

$2^{\mathrm{a}}$ ENCUESTA

$17 \% \quad(5)$

$66 \%(20)$

$17 \% \quad(5)$
Saben donde acudir ....20\% (6)

No saben donde acudir. . . 80\% (24)
93\% (28)

$7 \% \quad(2)$

$¿$ Crees que sexualidad yreproducción es lo mismo?

No ...........6.63\% (19)

Sí.............. 30\%

N.S.N.C.

$30 \%$

93\% (28)

$7 \% \quad(2)$

$0 \%$

Métodos anticonceptivos que conocen.

1 ó 2 métodos. ....... 63\% (19)

$30 \%(9)$

3 ó más métodos. . . . . 37\% (11)

$70 \%(21)$

Lugar para guardar un preservativo.

Lugar inadecuado . . . . 70\% (21)

$43 \%$ (13)

(cartera, bolsillo, etc.)

Lugar adecuado

(cajón mesita, etc.) . . . 30\% (9)

$57 \%(17)$

Cualquier sitio

donde los vendan . . . . . 60\% (18)

$0 \%$

Farmacia ......... 40\% (12)

$100 \%(30)$

Opinión de las chicas

que llevan preservativo

Opinan de forma positiva . 30\% (9)

Critican esta opción .... .70\% (21)

93\% (28)

$7 \% \quad(2)$

Qué hacer ante la ruptura

de un preservativo

No tienen claro qué hacer. $70 \%$ (21)

$27 \%$

$73 \%(22)$

Saben qué hacer....... 30\% (9)

Lubricantes que utilizarían

con el preservativo

Vaselina o aceites..... 90\% (27)

$37 \%(11)$

Lubricante del

preservativo $o$

especializados . . . . . 10\%

$63 \%(19)$

SIDA $\ldots \ldots \ldots \ldots .63 \%$ (25)

$67 \%(20)$

SIDA

y otras enfermedades ... 10\% (3)

$33 \%(10)$

N.S./N.C. . . . . . . . 7\%

(2)

$0 \%$

Vías de transmisión de las E.T.S.

Tienen una idea

aproximada .........10\% (3)

$73 \%(22)$

Penetración ........ . 70\% (20)

$20 \% \quad(6)$

N.S.N.C. . . . . . . 20. 20\% (6)

$7 \% \quad(2)$ 


\section{CUESTIONARIO ¿QUE SABES SOBRE SEXUALIDAD?}

1.- ¿Qué es para ti la sexualidad?

2.- ¿Crees que la sexualidad tiene algo que ver con la salud?

3.- ¿Con qué frecuencia hablas de sexualidad y con qué personas?

4.- ¿Dónde irías en tu ciudad para informarte sobre sexualidad y métodos anticonceptivos?

5.- ¿Crees que sexualidad y reproducción es lo mismo?

6.- ¿Qué métodos anticonceptivos conoces?

7.- ¿Dónde guardarías un preservativo?

8.- ¿Dónde comprarías los preservativos?

9.- ¿Qué opinas de las chicas que llevan un preservativo?

10.- ¿Qué harías si durante una relación sexual se rompiese el preservativo?

11.- ¿Qué tipo de lubricantes utilizarías al usar un preservativo?

12.- ¿Qué enfermedades de transmisión sexual conoces?

13.- ¿En qué tipo de contactos sexuales crees que se transmiten las enfermedades de transmisión sexual?

\section{DISCUSIÓN}

Observando los resultados obtenidos, vemos que ha habido una incidencia significativa de éxito, pero esto puede que no sea del todo cierto a largo plazo. Somos conscientes de que para producir un cambio en las actitudes de los educandos, requeriría un seguimiento posterior con refuerzo de los conocimientos tratados, aunque desafortunadamente, por el momento, no está dentro de las posibilidades de este estudio.

Aunque la pedagogía que hemos utilizado está basada en el pensamiento sociocrítico, probablemente nuestro estudio se haya estancado en el paradigma hermenéutico debido a las limitaciones de tiempo con las que contábamos. No podíamos disponer de muchas oportunidades para trabajar con la muestra, puesto que hay que tener en cuenta que nuestras charlas no estaban dentro del programa del curso escolar de los alumnos. De todos modos, no descartamos la posibilidad de hacer un seguimiento posterior.

Hemos tratado también de convertir a los propios educandos en transmisores de conocimientos a sus compañeros, de modo que el alcance de la información proporcionada no se limitase tan sólo a una muestra de 30 alumnos, sino a éstos y a los amigos de éstos, y a su vez, a los amigos de estos últimos.

Nuestra charla tenía también la intención de hacer que los alumnos tomasen conciencia de sus propios actos y de este modo, y ayudándose de los conocimientos administrados, inducir un cambio de actitud en su conducta. Pero lo fundamental es que fuesen ellos los dueños de sus acciones, que tomasen las riendas para que los cambios logrados sean duraderos en el tiempo. Son ellos los que han de decidir de que manera quieren actuar, pero disponiendo de la información pertinente.

Nos apoyamos también en la narración de la experiencia personal de embarazo no programado de una de nuestras compañeras, puesto que al tratarse de una persona cualificada para transmitir lo que significa un hecho de tal envergadura, podía implicar mejor a los alumnos en esta problemática y así reforzar la toma de conciencia.

Esperamos que en efecto, la toma de conciencia tenga cambio de actitud en la conducta, aunque somos conscientes de la necesidad de hacer un seguimiento para comprobarlo.

\section{CONCLUSIONES}

Nos ha parecido especialmente significativa la diferencia existente en los alumnos entre la primera y la segunda encuesta en aspectos como:

- Qué es la sexualidad.

- Dónde acudir para informarnos sobre sexualidad.

- Diferencia entre sexualidad y reproducción.

- Lugar donde comprar preservativos.

- Opinión de las chicas que llevan preservativos.

- Qué hacer ante la ruptura de un preservativo.

- Utilización de lubricantes con preservativos.

- Vías de transmisión de las E.T.S. 
Observando los resultados obtenidos se podría concluir que los alumnos han adquirido bastantes conocimientos en comparación con los que antes tenían. De este modo, consideramos que la hipótesis que formulábamos en la introducción se ha cumplido.

Además, consideramos que lo más interesante de este estudio ha sido dar la oportunidad a los alumnos de expresar sus sentimientos y temores en un debate. En esta ocasión fue donde realmente conocimos las dudas y miedos que tenían, al tiempo que tratábamos de solucionarlas. El encuentro terminó por parecerse más a un coloquio entre amigos que a un debate entre ponentes y alumnos.

\section{AGRADECIMIIENTOS}

Nuestro más sincero agradecimiento a los treinta jóvenes que decidieron participar gustosos en nuestro estudio, y que confiaron en nosotros sus sentimientos y temores más íntimos, haciendo de esta experiencia algo inolvidable e irrepetible.

Gracias a $\mathrm{M}^{\mathrm{a}}$ Carmen Botella y a Ana $\mathrm{M}^{\mathrm{a}}$ Sancho por brindarnos la oportunidad de trabajar con adolescentes, y a la directora del I.E.S. Leonardo Da Vinci, Silvia Gomis, por abrirnos las puertas de su centro.
En especial, muchas gracias a José Siles por mostrarnos su apoyo con paciencia infinita y por introducirnos en este apasionante mundo, que aunque nos ha robado gran parte de nuestro tiempo, nos ha resultado gratificante con creces.

\section{BIBLIOGRAFÍA}

Flitner, W. (1972), Manual de pedagogía general. Herder. Barcelona.

Quintana Cabanas, J.M. (1993). Sociología de la educación. Dykinson. Madrid.

Petrus, A. et cols.(1997). Pedagogía social. Arial. Barcelona.

Maspero, F. (1979). La pedagogía Freinet por quienes la practican. 19 experiencias escolares. Laia. Barcelona.

Quintana Cabanas, J.M. (1994). Pedagogía social. Dykinson. Madrid.

Bisquerra Alzina, R. et cols (1998). Modelos de orientación e intervención psicopedagógica. Praxis. Barcelona.

Siles González, J. (1998) Epistemología y enfermería: por una fundamentación científica y profesional de la disciplina. Enfermería clínica, vol. 7, núm 4.

Siles González, J.; García Hernández, E. (1995). Las características de los paradigmas y su adecuación a la investigación en Enfermería. Enfermería científica, núm. 160-161.

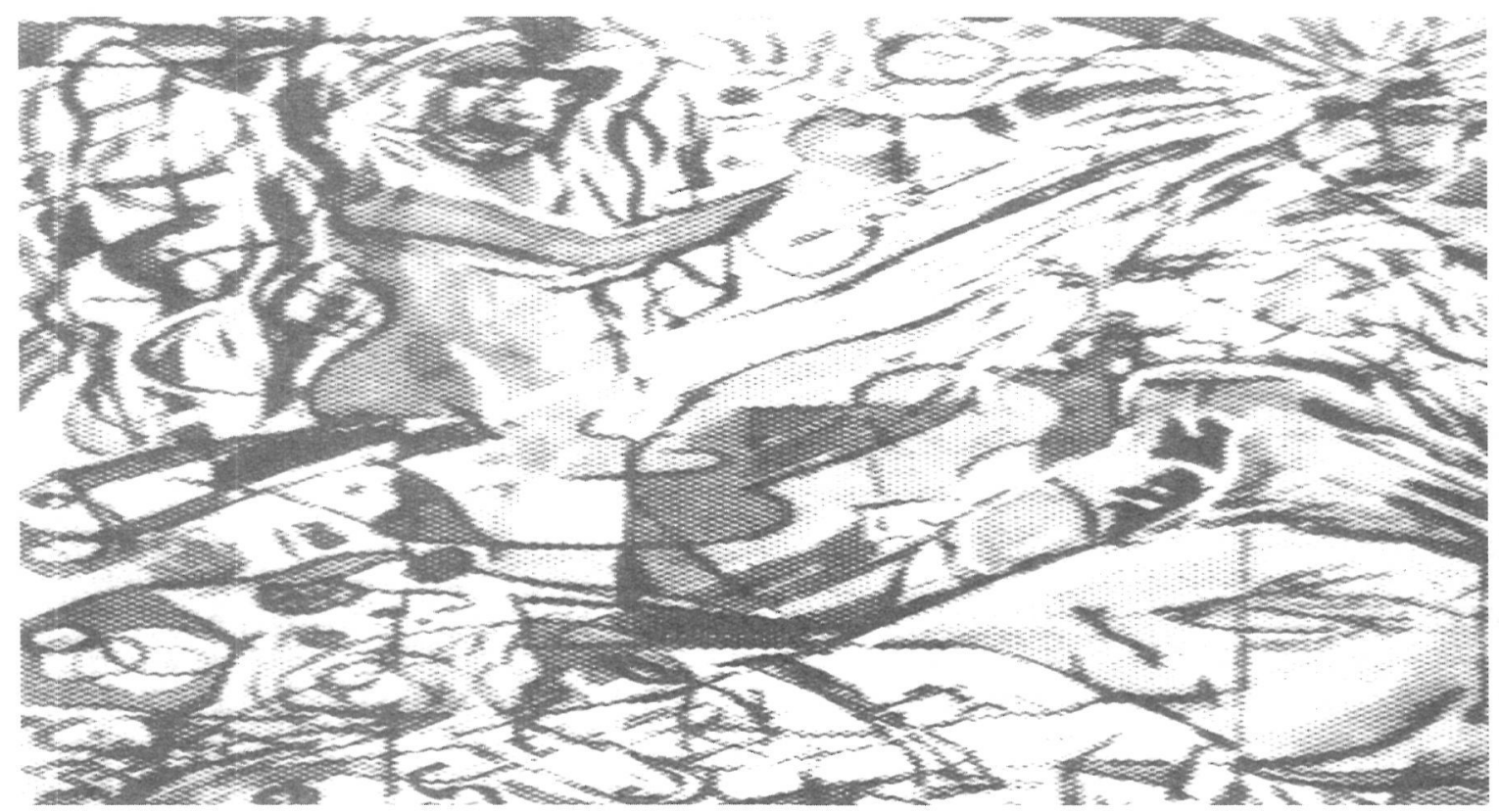

